\title{
An Improved Ant Colony Optimization for the Multi-Robot Path Planning with Timeliness
}

\author{
Shuai Zhou, GuangmingXiong ${ }^{*}$, Yong Li and Xiaoyun Li \\ Intelligent Vehicle Research Center,Beijing Institute of Technology and Key \\ Laboratory of Biomimetic Robots and Systems, Ministry of Education \\ 5 South Zhongguancun Street, Haidian District, Beijing, China
}

\begin{abstract}
To achieve efficient search performance for the multi-robot system which carries out the goal search task with consideration of timeliness, a multi-robot collaborative path planning system is designed to guide the robots during the search process. In the system, a planning method based on an Improved Ant Colony Optimization (IACQ) algorithm is proposed. In the solution procedure, the path cost and goal timeliness are taken as two optimization goals. Compared to the traditional optimization algorithm, the IACQ Algorithm has global superiority which brings about better solution Experiment results show that all robots accomplish the search task safely and efficiently by collabonative work using the proposed approach.
\end{abstract}

Keywords: multi-robot; collaborative path planning; ant colony optimization; timeliness

\section{Introduction}

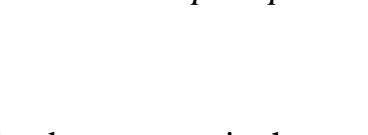

Task allocation in a multi-robot system s the problem of determining which robots should execute which tasks in prder lo achieve the overall system goals [1]. Many approaches are developed to solve this problem with optimization criteria such as travel distance or time [2, 3]. However, in some situation, we have to take the timeliness of the goal into consideration. Timeliness refers to the importance degree of goals, or the priority of goals. In some special tasks such as relief after disaster, emergency evacuation, the consideration of priority of goals is very important. As shown in Figure 1, a robot needs to visit the goals set $\{1,2,3,4,5\}$, and the black route is the shortest one. While in some special circumstances, goal 2 (red one) is in urgent and needs to be searched earlier, thus the red route is more suitable for the real situation than the black one in the search task. The route represented by the red line meets the timeliness requirement of the task with little sacrifice in path distance.
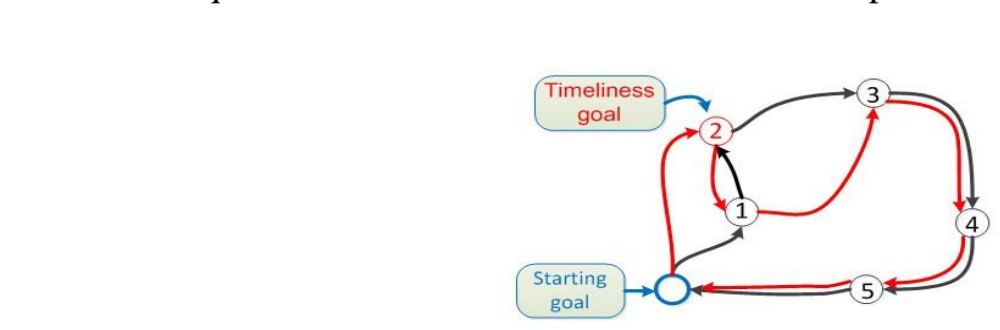

Figure 1.Goal Timeliness

\footnotetext{
* Corresponding Author
} 
In this paper, we focus on multi-goal search problem with timeliness for multiple robots. A team of robots at the same starting goal take task to search multiple goals known on the map and finally get back to the starting goal. It is similar to the classic Multi-Traveling Salesman Problem (MTSP) [4]. However, we not only consider about the distance factor, but also take the timeliness factor into consideration in this multi-goal search problem. Therefore, an Improved Ant Colony Optimization (IACO) algorithm is employed to solve the task allocation problem with consideration about shorter path as well as the timeliness requirement. Based on the proposed IACO algorithm, we design a multi-robot collaborative path planning system for search tasks with timeliness. The multi-robot system accomplishes the whole search task with high efficiency and quality through the mutual cooperation between robots.

The structure of the paper is as follows. In the second section, the architecture of the multirobot system is introduced. The planning method is proposed in the third section. Experimental results are presented in the section four. The last section draws a conclasion.

\section{System Architecture}

A hybrid multi-robot collaborative path planning system is designed to guide all robots to complete the search task. It contains three layers: task allocation layer, path planning layer and collaboration control layer as shown in Figure 2.

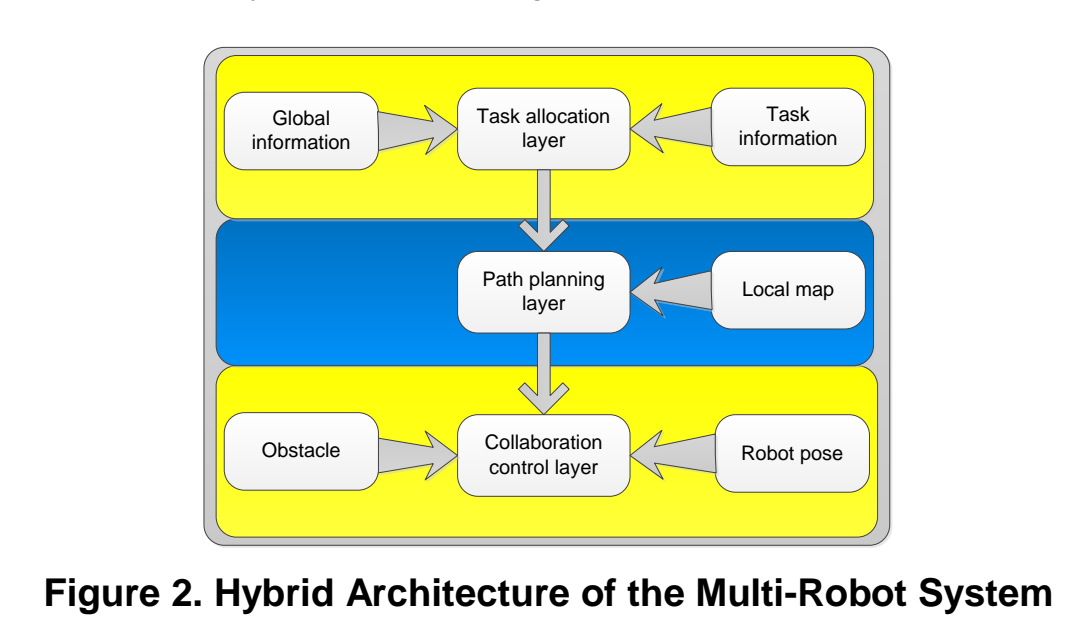

\subsection{Task Allocation Layer}

The task allocation layer is to allocate the task based on the planning information which contains the global environmental information as well as the information from the task itself. The globarenvironmental information refers to the map by which the task is carried out and the tas information refers to the positions and weights of all the goals in the search task. The search task is modeled as a MTSP and solved by the IACO algorithm. After this, the whole search task is divided to several sub-tasks for each robot to carry out. Each sub-task is a goal sequence that the corresponding robot needs to search one by one.

\subsection{Path Planning Layer}

Task allocated from the task allocation layer is a goal sequence connected by Euclidean line, not real path that the robot can track. In the IACO solving process, the path between two goals is assumed as Euclidean path, while it's not applicable to the real situation because of some obstacles. Thus paths between goals need to be planned in the path planning layer. A* 
algorithm is a heuristic search method which is widely used in the path search between two points on the map. And we employ it to plan the path between the two goals collected in the goal sequence. After this, we change the goal sequence into a route that the robot can track to complete its search task.

\subsection{Collaboration Control Layer}

All robots track their own route planned out from the path planning layer to complete their own search tasks in the task environment. The path planning layer considers the collision between robot and obstacle. During the process of the search task, collision may occur among robots. Collaboration control layer deals with this problem. It controls a robot to track its route, avoiding collision with other robots at the same time. Every robot sends its pose information to the 'central robot' constantly. There is a 'Blackboard' in the 'Central Robot' used to save the real time pose information with which the system can predict collisions inside the system. One robot slows down and another keeps moving when a collision perhaps occur between them. Using this collaborative method, all robots can complete the search task safely.

\section{Algorithm}

\subsection{Mathematical Model}

Goals with timeliness search problem studed in this paper can be called as a MTSP which is described as follows. There are $n$ goals (starting goalexcepted) $N=\left\{n_{1}, n_{2}, \cdots, n_{n}\right\}$ on the known map with corresponding weights $\varepsilon=\left\{\varepsilon_{1}, \varepsilon_{2}, \varepsilon_{n}\right\}$, and the position of each goal is given, distance from goal $i$ to $\backslash$ goal is $d\left(n_{i}, c\right)$. The multi-robot system consists of $m$ robots $M=\left\{m_{1}, m_{2}, \cdots, m_{m}\right\}$ which take tasks to visit all goals on the map. The solutions are m closed routes $R=\{r 1, r 2, r m\}$ which make sure that all goals in $N$ visited and visited only once, wherein $r i=n_{i 1}, n_{i 2}, \cdots, n$ represents the goal set consists of $i k$ goals that robot $m_{i}$ needs to search To improve the efficiency and quality of the search task, we add in consideration of the goal timeliness in the optimization solution procedure. According to the proposed search problem in this paper, two optimization goals need to be quantified: the total path distance (path cost) and the total waiting times of the goals with timeliness (timeliness cost).

Accurate path cost between two goals is unknown before the path is planned out, but we can use the Euclidean distance instead in the optimization solution procedure. The total path costs are the sum of all robots' path cost, calculated by Eq. (1).

$$
L_{\mathrm{R}}=\sum_{i=1}^{m} \sum_{j=1}^{i k} d\left(n_{i j}, n_{i(j+1)}\right) \quad\left(n_{i j} \in r i\right)
$$

where $r i$ represents the goals set consists of $i k$ goals that robot $m_{i}$ needs to search.

The timeliness cost of each goal with timeliness is the product of its waiting time and its goal weight. The waiting time can be estimated by its path cost from the starting goal and the velocity of the robot. Goal weight represents the urgency degree of the goal in the search task, and higher weight value should be assigned to the more urgent goal based on the situation. Total timeliness costs are the sum of all goals' timeliness cost, calculated by Eq. (2). 


$$
\mathrm{T}_{\mathrm{R}}=\sum_{i=1}^{m} \sum \varepsilon_{j} \times t\left(n_{\mathrm{ij}}\right) \quad\left(n_{i j} \in r i\right)
$$

For the goal without timeliness requirement, its weight value $\varepsilon$ is 0 and its time cost is also 0 . Higher total timeliness costs of the entire search task represent that the planning result is worse for the timeliness requirement and re-planning is a must. There are some constraints for this mathematical model:
(a) $\lfloor n / m\rfloor \leq i k \leq\lceil n / m\rceil$
(Balance search workload for each robot.)
(b) $r_{1} \cup r_{2} \cup \cdots \cup r_{m}=N$ (All goals should be searched.)
(c) $r_{i} \cap r_{j}=\varnothing \forall r_{i}, r_{j} \in R$ (Each goal should be searched only once.)

\subsection{IACO Algorithm}

The ability of real ants to find short paths between their nest and food sources s based on the indirect communication by the use of pheromones. This behavior of ant colonies has motivated the design of the Ant Colony Optimization (ACO) algorithm [5]. L was proved that the ACO has efficiency performance in NP hard coniomatorial optimization problem such as Travelling Salesman Problem (TSP) and Quadratic Assignment Probleñ (QAP) [6].

Traditional ACO algorithm only takes the path costs as pheromone factor when dealing with MTSP, in this paper, we take timeliness costs as another pheromone factor into the solution procedure. Based on new pheromone factors, an IACO algorithm is proposed to solve this problem. Table 1 gives out the pseudo code of the proposed IACO algorithm.

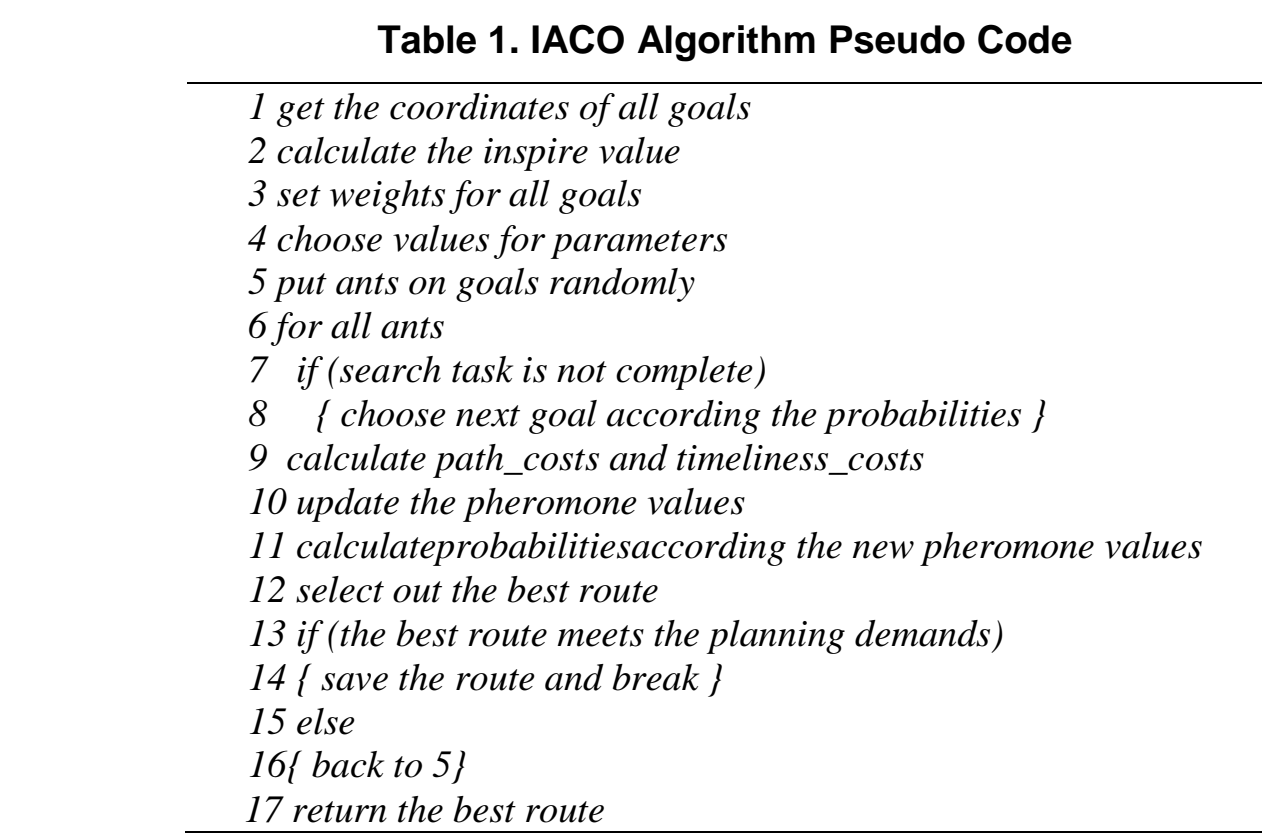

In the IACO algorithm, ants choose goal from the goal set consist of goals unsearched by the probabilities repeatedly until all goals get searched (step 8 in Table 1). Probabilities of the unsearched goals calculated in Eq. (3) are decided by the heuristic value $\eta$ and pheromone value $\tau$ corresponding to the goal. 


$$
p_{i j}^{k}(t)=\frac{\left[\tau_{i j}(t)\right]^{\alpha}\left[\eta_{i j}(t)\right]^{\beta}}{\sum_{l \in N_{i}^{k}(t)}\left[\tau_{i l}(t)\right]^{\alpha}\left[\eta_{i l}(t)\right]^{\beta}} \quad j \in N_{i}^{k}(t)
$$

where $p_{i j}^{k}(t)$ is the probability of ant $k$ moves from goal $i$ to goal $j$ in the $t$ times iteration process, and goal $j$ is form ${ }_{v_{i}}{ }^{k}(t)$ which is the unsearched goal set of ant $k ; \eta_{i j}(t)$ is the heuristic value for ant moving from goal $i$ to goal $j$, set as $1 / d\left(n_{i}, n_{j}\right)$, the inverse of the distance between the two goals; $\tau_{i j}(t)$ is the pheromone value for ant moving from goal $i$ to goal $j$, related to the whole solution.

After one iteration process, each ant's traversal route is a solution for the goals with timeliness search problem studied. For Ant-cycle Model adopted in the proposed IACO, after all ants have traversed all goals (an iterative process), pheromone value of each pat needs to be updated (step 10 in Table 1). The ants with excellent solutions spray more pheromone on the path causing more ants choose this path later which forms a positive eedback effect. The pheromone value updated through Eq. $(4-6)$. In Eq. (4) $\left.\Delta \tau_{i j}\right)(t)$ is the pheromone value sprayed on the path from goal $i$ to goal $j$ by ant $k$ after the $t$ times iteration process; $L_{k}$ is the total path cost of the search route planned by ant $k$ calculated in Eq. (1) and $T_{k}$ is the total time cost calculated in Eq. (2); $Q$ is constant which stands for the pheromone value; $q$ is the weight coefficient used to adjust the two factors' (path costs and timeliness costs) influence on the final solution.

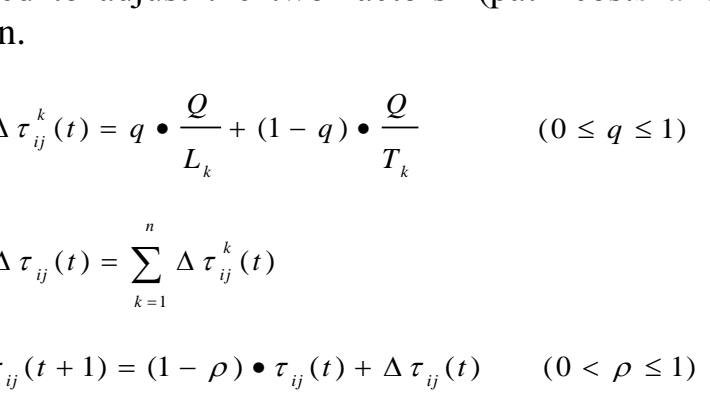

The total pheromone increnent on the path from goal $i$ to goal $j$ produced by all the $n$ ants after the iteration process is calculated by Eq. (5). Before the next iteration process, pheromone values for all paths on the route get updated by Eq. (6), wherein $\rho$ is the pheromone evaporation parameter. Repeat this iteration and update process until get the optimal solution.

\section{Experiment}

\subsection{Algorithm Test}

Compared with the traditional optimization method, IACO algorithm has the merits of global superiority and ability to obtain better solution. In the simulation experiment, 3 robots need to search 30 goals at the speed of $1(\mathrm{~m} / \mathrm{s})$ in a $10(\mathrm{~km}) * 10(\mathrm{~km})$ district. The solution results in total costs (path costs and timeliness costs) of the proposed IACO with the traditional Genetic Algorithm (GA) are given in Table 2. 
Table 2. Comparison between IACO and GA in Total Costs

\begin{tabular}{|c|c|c|c|c|c|}
\hline \multirow{2}{*}{$\begin{array}{l}\text { Goals } \\
\text { number }\end{array}$} & \multirow{2}{*}{$\begin{array}{c}\text { Timeliness } \\
\text { goals } \\
\text { number }\end{array}$} & \multicolumn{2}{|c|}{ Costs (IACO) } & \multicolumn{2}{|c|}{ Costs (GA) } \\
\hline & & $\begin{array}{c}\text { Path } \\
\text { costs }(\mathrm{km})\end{array}$ & $\begin{array}{l}\text { Timeliness } \\
\text { costs(min) }\end{array}$ & $\begin{array}{c}\text { Path } \\
\text { costs }(\mathrm{km})\end{array}$ & $\begin{array}{l}\text { Timeliness } \\
\text { costs(min) }\end{array}$ \\
\hline \multirow{3}{*}{30} & 0 & 55.45 & 0 & 56.78 & 0 \\
\hline & 5 & 58.50 & 11.62 & 57.42 & 22.54 \\
\hline & 10 & 65.36 & 65.33 & 72.91 & 78.59 \\
\hline
\end{tabular}

In Table 2, we can see that compared to GA, the IACO obtains better solution (less costs) which improves the efficiency of the multi-robot system dealing with the goals with timeliness search problem. In this experiment, we take the path costs the same important as the timeliness costs so that the weight coefficient value $q$ is set to 0.5 .

Interplay exists between the two optimization goals (path costs and timeliness costs) that makes it's not a Pareto Optimality [7] problem. But we can adjust the planjing result through changing the weight coefficient value $q$. The value of determines the planning result is more prone to lower path costs or lower timeliness costs (in Eq 4). As shown in Figure 3, with smaller $q$ value, the timeliness costs of the result is lower (blue line) and the path costs of the result is higher (red line), while with larger $q$ value, the situation is reversed. For both lower path costs and lower time costs at the same time, we set $q$ in the range $[0.4,0.6]$ in most situations.

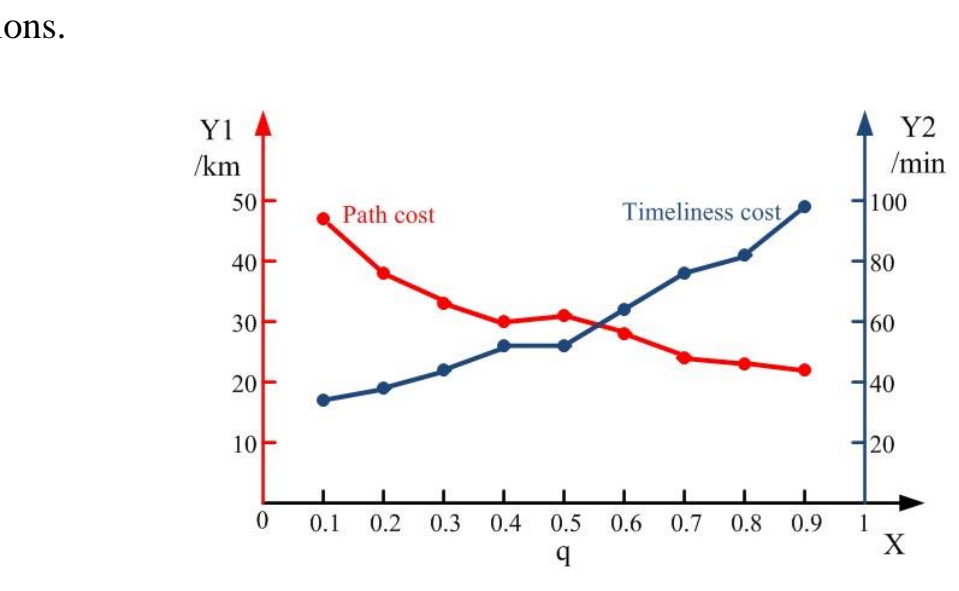

Figure 3. Influence of $q$ on the Planning Result

\subsection{Multi-robot System Test}

To test the performance of the proposed multi-robot collaborative path planning system in this paper, simulation and real robots experiments are conducted under the Player/Stage platform [8].

In the experiment, there are 16 goals and 3 of them $\{3,6,12\}$ with timeliness requirements need to search as shown in Figure 4. Timeliness goals need to be searched earlier relatively. The multi-robot system including 3 robots \{Robot1, Robot2, Robot3\} takes this search task. Robot1 is the 'Central Robot'. There are three linear obstacles. It is a multi-robot multi-goal path planning problem mentioned in the third section.

In task allocation layer, the 'Central Robot'- Robot 1 needs to allocate this task to other robots based on the planning information. The total search task is allocated by the proposed 
IACO algorithm and the search route of each robot is generated in the path planning layer. Figure 4 shows the planning result. The red line is Robot1's search route and it covers 5 goals $\{4,3,2,1,16\}$. The blue line is Robot2's search route and it also covers 5 goals $\{5,6,7,8,9\}$. The black line is Robot3's search route and it covers 6 goals $\{12,15,14,13,11,10\}$.
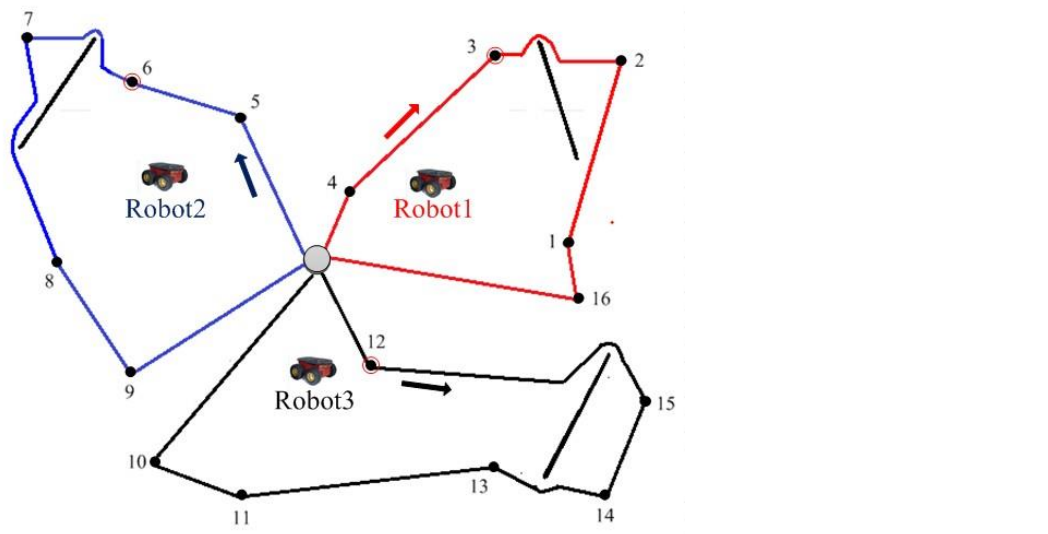

Figure 4. Planning Result

After all routes have been generated, all robots start to track them to carry out the search task in the collaboration control layer. Figure $5(\mathrm{a}-\mathrm{c})$ shows the simulation scenes in Stage, Figure 5(d-f) shows the real Pioneer3-AT robots experiments scenes. In Figure 5, (a) and (d) are the beginning period of the search task, (b) and (e) at the middle period of the search task; (c) and (f) are the ending period of the search task. In the whole search process, all robots can avoid collision with obstacles and other robots.

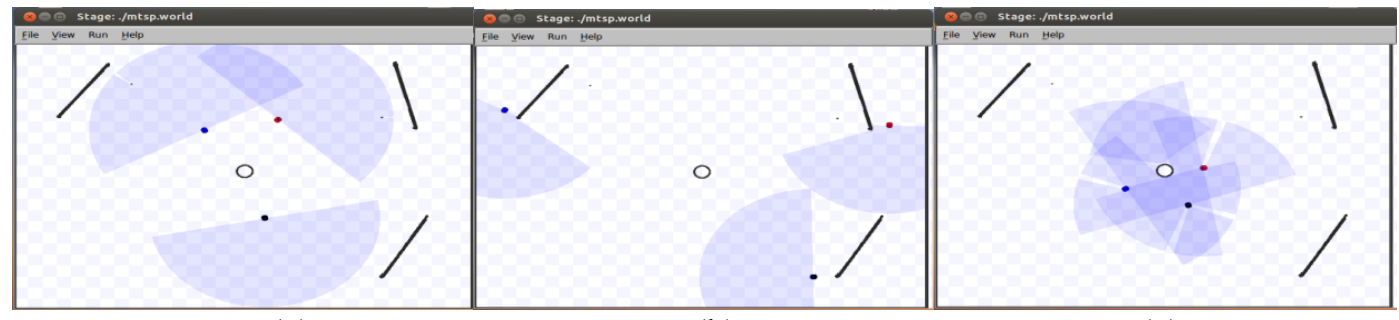

(a)

(b)

(c)

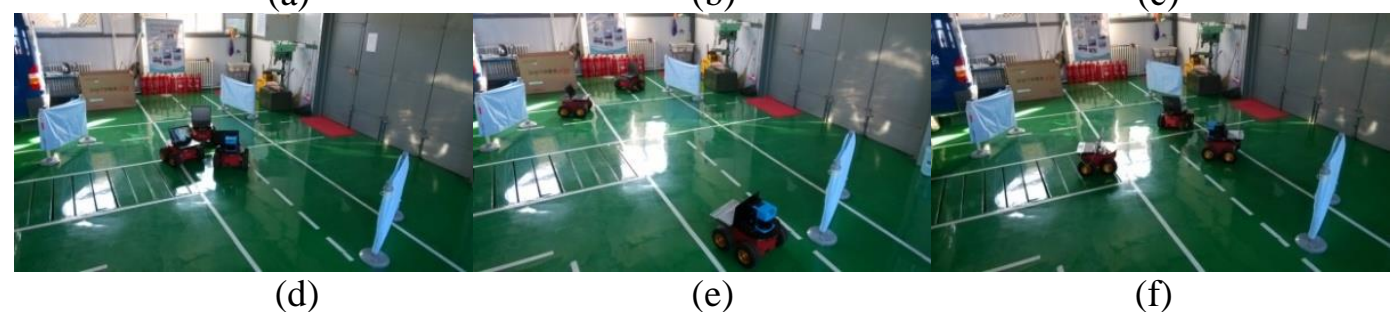

Figure 5. Experiment Scenes

\section{Conclusion}

A multi-robot collaborative path planning system was designed in this paper. To solve the search problem, an IACO (Improved Ant Colony Optimization) algorithm was proposed. The comparison result with traditional algorithm illustrated that the method proposed in this paper is superior. Simulation and real robots experiments were conducted to test the performance of 
the system and the results showed that the multi-robot collaborative path planning system proposed in this paper can accomplish the goals with timeliness search task in high efficiency and stability.

\section{Acknowledgements}

This research was partially supported by the National Natural Science Foundation of China (Grant No. 91120015).

\section{References}

[1] G. AyorkorKorsah, A. Stentz and M. Bernardine Dias, "A comprehensive taxonomy for multi-robot task allocation", The International Journal of Robotics Research, (2013), pp. 1495-1513.

[2] Z. Xin, G. Gilles and D. Rémy, "A genetic approach to solving the vehicle routing problem with tumedependent travel times", Mediterranean Conference on Control and Automation - Conference Proceedings. Piscataway, NJ 08855-1331, United States: Inst. of Elec. and Elec. Eng. Computer Sociery, (2008), pp. 413418.

[3] J. Miller, K. Sunil and T. Menard, "Intelligent Transportation Systems Trâveling Salesman Problem (ITSTSP) -A Specialized TSP with Dynamic Edge Weights and Intermediate Cities", 13th International IEEE Conference on Intelligent Transportation Systems. Piscataway, NJ, USA: IEEE, (2010), pp. 992-997.

[4] C. Walter, M. T. S. P. Marques and M. M. Pacheco, "Verbal cues in motor learning focus on the component and on the interaction between components", Journal of sport \& exercise psychology. 1607 n market st, Po Box 5076, Champaign, il 61820-2200 USA: Human Kinetics PUBL INC, vol. 34, (2012), pp. s143-s143.

[5] M. Dorigo and G. Di Caro, "The ant colony optimization meta-heuristic", New Ideas in Optimization, D. Corne, M. Dorigo and F. Glover, Eds. New York: McGraw-Hill, (1999), pp. 11-32.

[6] M. Dorigo, V. Maniezzo and A. Colorni, "The Ant System: Optimization by a colony of cooperating agents", IEEE Trans. On Systems, Man and Cybernetics-Part B, vol. 26, no 1, (1996), pp. 1-3.

[7] E. Zitzler and L. Thiele, "Multi-objective Evolutionary Algorithms", A Comparative Case Study and the Strength Pareto Approach, IEEE Transactions on Evolutionary Computation, vol. 3, no. 4, (1999), pp. 257271.

[8] B. P. Gerkey, R. T. Vaughan and A. Howard, "The Player / Stage Project: Tools for Multi-Robot and Distributed Sensor Systems", Proceedings of the International Conference on Advanced Robotics (ICAR 2003), (2003), pp. 317 - 323.

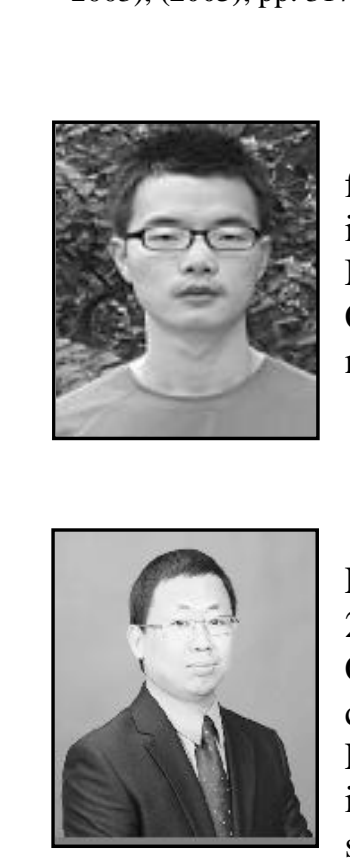

Guangming Xiong received the Ph.D. degree in Mechanical Engineering from Beijing Institute of Technology, Beijing, China, in 2005.He was a Visiting Scholar with the Department of Electrical and Computer Engineering, Brigham Young University, Provo, USA. He is currently an Associate Professor with the School of Mechanical Engineering, Beijing Institute of Technology. His research interests include planning and control for intelligent vehicles and multi-robot system, human-machine interface and multi-vehicle cooperation. 


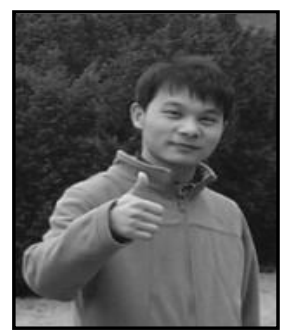

Yong Li received the B.E. degree in Vehicle Engineering from Hubei University of Automotive Technology, Shiyan, China, in 2012. He is currently working toward the M.Eng. degree with the School of Mechanical Engineering, Beijing Institute of Technology, Beijing, China. His research interests include planning and control for intelligent vehicles, multi-vehicle cooperation.

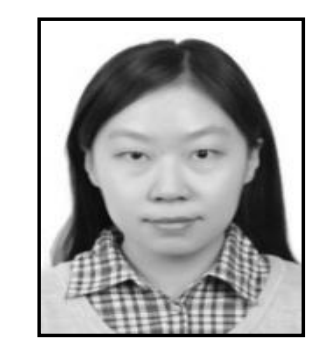

Xiaoyun Li received the B.E. degree in Safety Engineering from Beijing Institute of Technology, Beijing, China, in 2013. She is currently working toward the M.Eng. degree with the School of Mechanical Engineering, Beijing Institute of Technology Beijing, China. Her research interests include planning and control for multi-robot system.

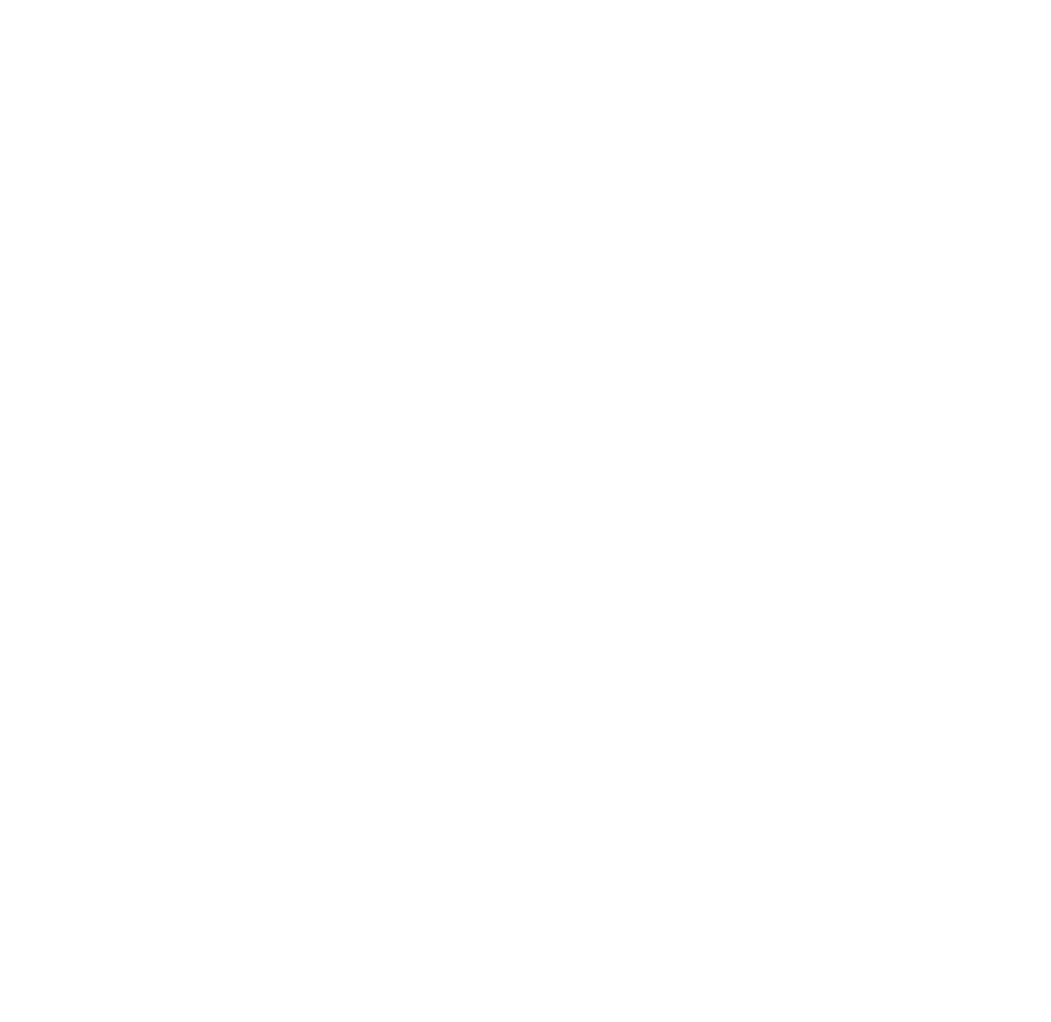


International Journal of Smart Home

Vol.8, No.2 (2014)

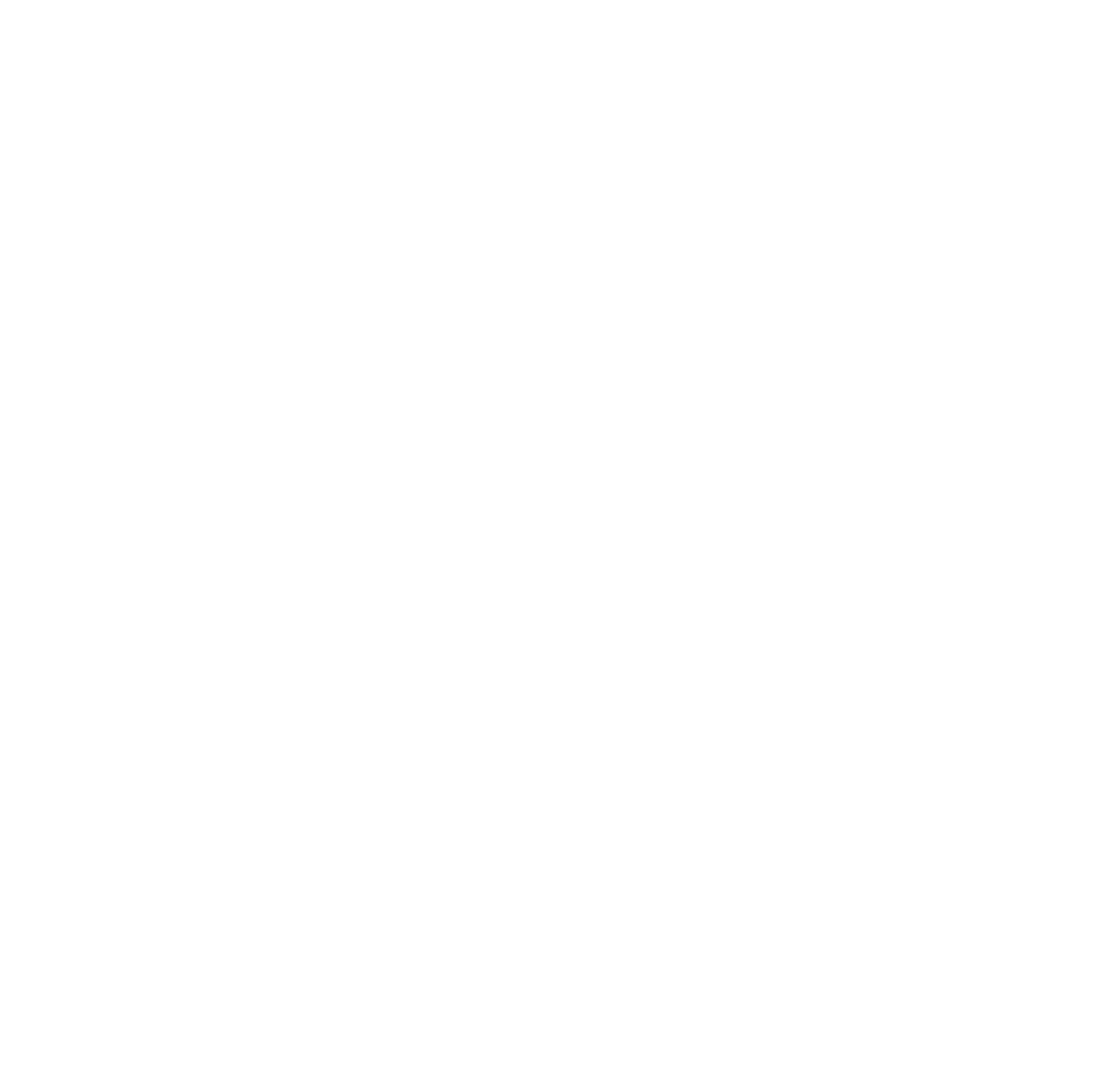

